\title{
Zum Abbau des Xanthins und Coffeins im Organismus des Menschen. \\ Von \\ Walter Levinthal.
}

(Aus der II. Medizinischen Klinik in München, Direktor Prof. F. v. Müller.)

(Der Redaktion zugegangen am 29. Februar 1912.)

Obwohl kaum ein Gebiet der physiologischen Chemie in den letzten Jahren nach den verschiedensten Seiten so mannigfaltig durchgearbeitet worden, wie das des Purinstoffwechsels, ist bis heute noch eine ganze Reihe selbst der hauptsächlichsten Probleme Gegenstand der Untersuchung und Diskussion. Ich erinnere nur an zwei Dinge: So wird 1. die Frage, ob wir die Harnsäure als unangreifbar im menschlichen Organismus aufzufassen haben, oder einen weiteren Abbau, etwa bis zum Harnstoff, annehmen dürfen, zwar von der Mehrzahl der Autoren (z. B. Wiechowski, Umber, Klemperer u. a. $\left.{ }^{1}\right)$ ) heute im ersteren Sinne beantwortet, aber trotzdem glauben einige Untersucher mit ihren Resultaten an dem alten Standpunkt vom Abbau festhalten zu müssen.2) Und 2. denke man an das Problem des Abbaues der methylierten Xanthine, im wesentlichen an die Frage der Entstehung von Harnsäure aus Coffein, Theobromin und Theophyllin. Ich bemerke, da $\mathrm{das}$ zuerst erwähnte Problem nicht zum Gegenstande dieser Arbeit gehört, daß sich aber Gelegenheit finden wird, zum zweiten Stellung zu nehmen.

Seit über 10 Jahren ist von den verschiedensten Seiten die Entstehung der Harnsäure, und zwar sowohl der endogenen, wie exogenen Komponente, Gegenstand der Untersuchung gebaden 1910 .

1) cf. Sitzungsbericht des Deutsch. Kongr. f. innere Medizin, Wies-

2) z. B. Frank und Schittenhelm, Diese Zeitschrift, Bd. 63, S. $269 \mathrm{ff}$. 
wesen. Ausgehend von der Anschauung, daß der exogene Anteil im wesentlichen, wenn nicht gar ausschließlich, aus den in der Nahrung präformierten Purinkörpern stamme, ist man daran gegangen, in möglichst exakten physiologischen Versuchen den Anteil der einzelnen Purinbasen festzustellen. So liegen heute eine ganze Reihe von Resultaten im besonderen für den menschlichen Organismus vor. ${ }^{1}$ )

Und da zeigt es sich, daß kaum zwei solcher Reihen bei den verschiedenen Autoren annähernde Übereinstimmung zeigen. Es sollte hier die Aufgabe gestellt werden, nachzusehen, ob es gelänge, eine Erklärung für die Verschiedenartigkeit dieser Resultate zu finden; und deshalb wurden die Untersuchungen auf einen einzigen Purinkörper, das Xanthin, beschränkt. Für die Wahl gerade dieser Base war des weiteren der Umstand maßgebend, daß gerade sie am wenigsten bisher untersucht worden, besonders im Vergleich zum Hypoxanthin weit zurücksteht. Schließlich aber darf das Xanthin unter sämtlichen Purinbasen eine besondere Stellung beanspruchen, weil es die letzte Vorstufe zur Harnsäure und damit nach unserer Anschauung eine obligatorische Durchgangsstation für den gesamten Purinstoffwechsel darstellt und überdies der Ausgangspunkt der methylierten Xanthine ist. So lautete also das Problem : Es war nachzusehen, in welcher Menge und in welcher Form nach verschiedenen Applikationsmethoden das Xanthin beim Menschen wiedergefunden werden könnte. Diese Versuche wären eventuell auch auf den Gichtkranken auszudehnen. Nicht zuletzt wurde an diese Untersuchung die Hoffnung geknüpft, daß es vielleicht gelänge, im Xanthin ein brauchbareres diagnostisches Hilfsmittel für die Gicht zu finden, als es die bisher gebräuchliche Thymus oder Nucleinsäure darstellt.

Ich gebe zuerst einige Zahlen aus der Literatur: Die Untersuchungen über Hypoxanthin und die Amidopurine reichen etwas weiter als die des Xanthins zurück. So fand Minkowski2) bereits 1898 bis $50 \%$ verfütterten Hypoxanthin-

$\left.{ }^{1}\right)$ Literaturübersicht bei Bloch, Biochem. Zentralbl., Bd. 5, S. $562 \mathrm{ff}$.

2) Minkowski, Archiv f. exper. Pathol. u. Pharmakol., Bd. 41, S. $375,1898$. 
stickstoffs als Harnsäure wieder, einen Befund, den Burian und Schur ${ }^{1}$ ) bald darauf bestätigen konnten. Dagegen hatten die älteren Arbeiten über Guanin (Kerner und Stadthagen, Burian und Schur) und Adenin (Kossel, Minkowski) nur zu negativen Resultaten geführt. ${ }^{2}$ )

Die ersten, die Xanthinversuche an Menschen unternommen haben, waren Krüger und Schmid in ihrer grundlegenden Arbeit aus dem Jahre 1902. ${ }^{3}$ ) Aus der Zeit vorher liegt nur ein Versuch am Hunde von Nencki und Sieber ${ }^{4}$ ) und einer am Kaninchen von Krüger und Salomon, ${ }^{5}$ ) beide mit negativem Erfolge, vor. Krüger und Schmid gelang es, von den verfütterten $1,5 \mathrm{~g}$ der Base mit $0,5520 \mathrm{~g} \mathrm{~N} \mathrm{10,2 \%}$ als Harnsäure und $1 \%$ im Basengemisch wiederzufinden. Erheblich größer stellt sich bei ihnen die Steigerung der Harnsäure nach Verfütterung von Hypoxanthin und Adenin dar, wieder ausgedrückt in Prozenten des verfütterten Purinstickstoffs :

$\begin{array}{cc}\text { Nach Hypoxanthin } & \text { ca. } 62 \% \\ \gg \text { Adenin } & \text { » } 41 \%\end{array}$

Die Steigerung des Basenstickstoffs ist viel kleiner, die stärkste tritt mit einem Werte von $3 \%$ nach Adenin auf.

Aus der Folgezeit stammen dann eine ganze Reihe von Untersuchungen über die Verfütterung von Hypoxanthin und von den Amidopurinen. Ich nenne nur noch Landau, ${ }^{6}$ ) der nach Gaben von 0,8 bis $1,5 \mathrm{~g}$ Hypoxanthin sogar 46 bis $88 \%$ wieder gewinnt, ferner Brugsch und Schittenhelm, ${ }^{7}$ ) deren Zahlen bei einem Gichtiker folgende sind:

1) Burian u. Schur, Archiv f. d. ges. Physiol., Bd. 87, S. 239, 1901. 1902.

2) Zitiert b. Krüger u. Schmid, Diese Zeitschrift, Bd. 34, S. 549 ff.,

3) Krüger u. Schmid, Diese Zeitschrift, Bd. 34, S. 549, 1902.

4) Nencki u. Sieber, Pflügers Archiv, Bd. 31, S. 347.

$\left.{ }^{5}\right)$ Krüger u. Salomon, Diese Zeitschrift, Bd. 31, S. 184 (Anm.).

$\left.{ }^{6}\right)$ Landau, Deutsch. Arch. f. klin. Med., Bd. 95, S. 280, 1909.

7) Brugsch u. Schittenhelm, Zeitschrift f. exp. Pathol., Bd. 5, S. 215, 1908. 


$\begin{array}{ll}\text { Adenin } & 50 \% \\ \text { Guanin } & \text { Spuren } \\ \text { Hypoxanthin } & 13 \%\end{array}$

und schließlich Rotky ${ }^{1}$ ), der Guanin- und Hypoxanthinversuche an Patienten verschiedener Art anstellt.

Angaben über Xanthinverfütterung finde ich erst wieder im Jahre 1910 bei Lafayette Mendel und John Lyman. ${ }^{2}$ ) Diese Autoren haben neben Versuchen am Kaninchen, Hund, Schwein den Purinstoffwechsel nach Verfütterung von Xanthin, Hypoxanthin, Adenin und Guanin an zwei stoffwechselgesunden Individuen untersucht. Ihre Resultate sind in der folgenden Zusammenstellung enthalten, wieder ausgedrückt in Prozenten des verfütterten Purinstickstoffs :

I. Fall: $\left\{\begin{array}{lll}\text { Hypoxanthin } & \overline{\mathrm{U}}-\mathrm{N} & \mathrm{B}-\mathrm{N} \\ \text { Xanthin } & 54 & 4 \\ \text { Guanin } & 33 & 2 \\ \text { Adenin } & 37 & 3 \\ \text { II. Fall: } & \left\{\begin{array}{lll}\text { Hypoxanthin } & 56,6 & 3,6 \\ \text { Xanthin } & 46 & 1 \\ \text { Guanin } & 19,5 & 2,7 \\ \text { Adenin } & 30 & 3\end{array}\right.\end{array}\right.$

Bei ihnen finde ich auch einen Fall von Plimmer, Dick und Li eb ${ }^{3}$ ) zitiert, wo die Erhöhung der Harnsäureausscheidung nach Xanthin und Guanin 10\% des verfütterten Purinstickstoffs nicht überschritt.

Das Präparat, das wir zu unsern Untersuchungen verwandten, stammte aus den Elberfelder Farbwerken und war uns vom Direktor Prof. Dr. Duisberg in dankenswertester Weise zur Verfügung gestellt. Da die Konservierung der freien Base auf mannigfaltige Schwierigkeiten stößt, so erhielten wir das Xanthin als $\mathrm{Na}-\mathrm{Salz}$ von der Formel $\mathrm{C}_{5} \mathrm{~N}_{4} \mathrm{H}_{3} \mathrm{O}_{2} \mathrm{Na}+\mathrm{H}_{8} \mathrm{O}$.

1) Rotky, Deutsch. Arch. f. klin. Med., Bd. 98, S. 540, 1910.

$\left.{ }^{2}\right)$ Mendel und Lyman, Journ. of biologic. Chemistr., Bd. 8, Nr.2, S. $115 \mathrm{ff}$., 1910 .

3) Plimmer, Dick u. Lieb, Journ. of Physiol., Bd. 39, S. 98, 1909. 
Da die Stickstoffbestimmung keinen völlig entsprechenden Wert ergab, so wurde eine genaue Analyse des Präparates auf dreifachem Wege unternommen. 1. Die Kupferfällung einer bestimmten in Wasser gelösten Menge nach Krüger-Schmid. 2. Die Reinigung des Präparates und die Gewinnung der freien Base nach Horbaczewski und 3. die Bestimmung des Silberresp. Silbernitratdoppelsalzes.

Die Berechnung nach der obigen Formel verlangt einen Xanthingehalt von $79 \%$, d. i. ein Stickstoffgehalt von $29 \%$.

1. Die Kupferfällung ergab ca. $65 \%$ Purinbase, d. i. ein Stickstoffgehalt von $24 \%$.

2. Der zweite Weg, bei dem in mehrfachen Kontrollen eine bestimmte Menge des Präparates aus seiner Lösung in $\mathrm{NaOH}$, die mit Wasser verdünnt war, durch Essigsäure gefällt wurde, ergab eine Ausbeute von ca. $59 \%$ reinen wasserfreien Xanthins. (Verlangt $36,84 \% \mathrm{~N}$, gefunden 36,81 bis $36,87 \%$ N.)

Ich bemerke, daß diese Reinigung des Präparates nach Horbaczewski anfangs auf Schwierigkeiten stieß; ich hätte sonst die Base immer erst nach ihrer Reingewinnung und jedesmaligen analytischen Identifizierung für meine Untersuchungen verwandt. So mußte die einmal begonnene Methode, das Präparat selbst $z u$ applizieren, beibehalten werden.

3. Der dritte Weg, die Gewinnung des Silbersalzes und weiterhin des Silbernitratsalzes, stieß auf die bekannten Schwierigkeiten. Ich erinnere daran, daß die Zusammensetzung dieser Salze in der Literatur inkl. den Lehrbüchern verschieden angegeben, eventuell als dubiös bezeichnet wird. Die Methode war folgende: Eine bestimmte Menge des Salzes wurde in $\mathrm{NH}_{3}$ gelöst, mit ammoniakalischer Silberlösung ausgefällt, nach mehrstündigem Stehen abgesaugt und mit ammoniakhaltigem Wasser bis zur völligen Chlor- und Silberreinheit gewaschen, im Brutapparat getrocknet. Das dabei meist schwarz werdende Salz wurde in Salpetersäure von der Dichte 1,1, d. i. 17,11\% ig, heiß gelöst. Beim Erkalten krystallisierte das Silbernitratsalz aus.

Die Analysen des Silbersalzes ergaben in 3 bis 4 Bestimmungen sehr schwankende Werte: Für N 16-18\%, für $\mathrm{Ag} 48-54 \%$.

Für $\mathrm{C}_{5} \mathrm{H}_{4} \mathrm{~N}_{4} \mathrm{O}_{2} \cdot \mathrm{Ag}_{2} \mathrm{O}$ berechnet sich: $\mathrm{N} 14,6 \%, \mathrm{Ag} 56,25 \%$

$\begin{array}{lllll}\gg \mathrm{C}_{5} \mathrm{H}_{4} \mathrm{~N}_{4} \mathrm{O}_{2} \cdot \mathrm{AgO} & \star & >20,3 \%, & >40,6 \% \\ \gg\left(\mathrm{C}_{5} \mathrm{H}_{4} \mathrm{~N}_{4} \mathrm{O}_{2}\right)_{2} \cdot \mathrm{Ag}_{2} \mathrm{O} & > & > & >21 \%, & >40 \% \\ >\left(\mathrm{C}_{5} \mathrm{H}_{4} \mathrm{~N}_{4} \mathrm{O}_{2}\right)_{2} \cdot \mathrm{AgO} & > & > & >26 \%, & >25 \%\end{array}$

Für keine dieser Formeln konnten die gefundenen Werte in befriedigende Übereinstimmung gesetzt werden. 
Das Silbernitratsalz wird meist aufgefaßt als eine einfache Addition des Xanthins mit Silbernitrat von der Formel $\mathrm{C}_{5} \mathrm{~N}_{4} \mathrm{H}_{4} \mathrm{O}_{2} \cdot \mathrm{AgNO}_{3}$. Ist diese Formel richtig, so verlangt sie einen Gehalt von $17,4 \% \mathrm{~N}$ und von $33,5 \% \mathrm{Ag}$. Die Bestimmung dieser Werte stößt nun deshalb auf Schwierigkeiten, weil, wie schon Strecker ${ }^{1}$ ) angibt, beim Waschen des Krystalles mit Wasser angeblich das Silber entfernt und zwar eventuell bis zur Hälfte reduziert wird. lch versuchte deshalb zu einem Ziele zu gelangen, indem ich die abgesaugten Krystalle zum Teil mit Wasser, zum Teil mit Äther wusch, zum Teil völlig ungewaschen ließ. In jedem Fall wurde eine Trocknung bei ca. 80 bis $130^{\circ}$ vorgenommen. Dabei ergaben sich auffallenderweise für alle 3 Fälle leidlich übereinstimmende Resultate, die sowohl was den N-Gehalt, wie den Ag-Gehalt betrifft, für die oben angegebene Formel nicht stimmten. Mikroskopisch zeigten sich überall zierliche Büschel feiner prismatischer Nadeln. Da die Trocknung bei 130 bis $140^{\circ}$ noch einen Wasserverlust von $3,62 \%$ ergab, so gelingt es, sämtliche Werte in genügende Übereinstimmung zu setzen, wenn man die Formel $\left(\mathbf{C}_{5} \mathbf{H}_{4} \mathbf{N}_{4} \mathbf{O}_{\mathbf{2}}\right)_{\mathbf{2}} \cdot \mathbf{A g N O} \mathbf{N}_{3}$ $+\mathbf{H}_{2} \mathbf{O}$ für die Krystalle annimmt. Hier meine Zahlen:

$\mathrm{N}$

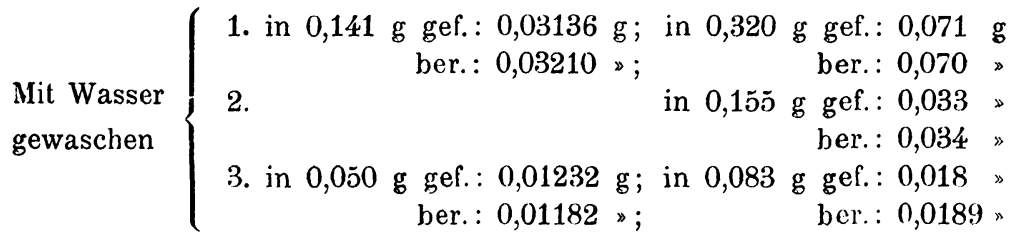

4. bei $75^{\circ}$ getrocknet in $0,031 \mathrm{~g}$ gef.: $0,007 \tilde{6} \mathrm{~g}$ ber. : 0,00707 bei $1.40^{\circ}$ weitergelrocknet Verlust von $6 \% \mathrm{H}_{2} \mathrm{O}$, davon

gewaschen in $0,039 \mathrm{~g}$ gef.: $0,0099 \mathrm{~g}$; in $0,070 \mathrm{~g}$ gef. : $0,017 \mathrm{~g}$ ber.: 0,0092 ; ber.: 0,016

Ungewaschen : 5 . in 0,052 g gef.: $0,01246 \mathrm{~g}$; in $0,112 \mathrm{~g}$ gef.: $0,023 \mathrm{~g}$ ber. : 0,0114 >; ber.: 0,025 》

6. Wasserverlust bei Trocknung von $0,138 \mathrm{~g}$ bei 130 bis $140^{\circ}: 0,00$ 气 $\mathrm{g}=3,62 \%$; berechnet: $3,66^{\circ} \%$.

Da eine quantitative Auskrystallisation aus der essigsauren Lösung kaum erwartet werden konnte, darf angenommen werden, daß unser Präparat ca. $65 \%$ Xanthin mit $24 \% \mathrm{~N}$ bei einer Verunreinigung von ca. $14 \%$ enthält. Diese Zahlen sollen allen Berechnungen zugrunde gelegt werden.

1) Strecker, Liebigs Annalen, Bd. 108, S. 146. 
Alle Zahlen sind Mittelwerte von Doppelbestimmungen, die für Harnsäure-Basen im Urin nach Krüger-Schmid, für die Basen in den Faeces nach Krüger-Schittenhelm, für den Stickstoff nach Kjeldahl gewonnen wurden.

Die erste Versuchsreihe wurde von einem stoffwechselgesunden Manne E. N. von 27 Jahren mit einem Körpergewicht von $64,5 \mathrm{~kg}$ bei purinfreier Diät erhalten. Die folgenden Tabellen I und II geben die Werte 1. nach einer Darreichung von $300 \mathrm{~g}$ Thymus, 2. von $4,1 \mathrm{~g}$ unseres Xanthinpräparates in wässeriger Lösung per os. Leider konnten die Versuche nicht fortgesetzt werden, da das betreffende Versuchsindividuum nicht zu bewegen war, die Kost weiterhin einzuhalten.

Tabelle I. E. N.

\begin{tabular}{c|c|c|c|c|c|c}
\hline $\begin{array}{c}\text { Datum } \\
1911\end{array}$ & $\begin{array}{c}\text { Harnmenge } \\
\text { in ccm }\end{array}$ & Spez.Gew. & $\begin{array}{c}\text { Ges.-N } \\
\text { in g }\end{array}$ & $\begin{array}{c}\overline{\mathrm{U}}-\mathrm{N} \\
\text { in } \mathrm{g}\end{array}$ & $\begin{array}{c}\mathrm{B}-\mathrm{N} \\
\text { in } \mathrm{g}\end{array}$ & \\
\hline 31. I. & 800 & 1025 & 7,8 & 0,1540 & 0,0101 & \\
1. II. & 1160 & 1016 & 8,7 & 0,1380 & 0,0114 & \\
2. & 1300 & 1017 & 9,0 & 0,1397 & 0,0109 & \\
4. & 1000 & 1016 & 7,2 & 0,1285 & 0,0074 & \\
\hline Mittel vom 31. I.-4. II. . . & 8,2 & 0,1400 & 0,0100 & 300 g Thy m us mit \\
einem Purin-N-Gehalt \\
5. II.
\end{tabular}

das ist eine Steigerung von $0,097 \mathrm{~g} \overline{\mathrm{U}}-\mathrm{N}$ und von $0,003 \mathrm{~g}$ B-N in Summa $0,100 \mathrm{~g}=\mathbf{8 , 3} \%$ der verfütterten N-Menge.

Es zeigt sich also, daß in diesem Falle die Erhöhung des Purinstickstoffes nach der Thymusdarreichung außerordentlich gering ist; dem entspricht die relativ dürftige Ausbeute nach dem Xanthin. Der Vergleich mit den folgenden Versuchen zwingt zu der Annahme, daß die Resorptionsbedingungen bei E. N. ziemlich ungünstige gewesen sind.

Meine Hauptresultate gewann ich an Selbstversuchen, wobei ich vom 20. Januar bis zum 2. April und vom 1. Juni bis zum 4. Juli 1911 auf purinfreier Diät hielt. Ich habe nie- 
mals irgend welche Symptome einer Stoffwechsel- oder Nierenkrankheit gezeigt. Der Urin blieb in der Untersuchungszeit auch an den Xanthintagen frei von Albumen. Die Kost wurde anfangs tadellos, später nur mit Mühe ertragen, worauf wohl das geringe Absinken der Normalwerte gegen Ende der Kurve zu beziehen sein dürfte. Der Gesamtgewichtsverlust in der ersten dreimonatlichen Periode betrug übrigens nur $4 \mathrm{~kg}$.

Tabelle II: E. N.

\begin{tabular}{|c|c|c|c|c|c|c|}
\hline $\begin{array}{c}\text { Datum } \\
1911\end{array}$ & $\begin{array}{c}\text { Harnmenge } \\
\text { in } \mathrm{ccm}\end{array}$ & Spez.Gew. & $\left|\begin{array}{c}\text { Ges. }-\mathrm{N} \\
\text { in } \mathrm{g}\end{array}\right|$ & $\begin{array}{l}\overline{\mathrm{U}}-\mathrm{N} \\
\text { in } \mathrm{g}\end{array}$ & $\begin{array}{l}\mathrm{B}-\mathrm{N} \\
\text { in } \mathrm{g}\end{array}$ & \\
\hline 10. II. & 1200 & 1016 & 7,0 & 0,1604 & 0,0147 & \\
\hline 11. & 900 & 1020 & 6,3 & 0,1364 & 0,0132 & \\
\hline 12. & 1060 & 1018 & 7,8 & 0,1490 & 0,0137 & \\
\hline 14. & 680 & 1024 & 7,0 & 0,1571 & 0,0105 & \\
\hline \multicolumn{3}{|c|}{ Mittel vom 10.-14. II. . • • } & 7,0 & 0,1507 & 0,013 & $\begin{array}{l}4,1 \mathrm{~g} \text { unseres Xanthin- } \\
\text { präparates mit } 0,98 \mathrm{~g} \mathrm{~N}\end{array}$ \\
\hline 15. II. & 1360 & 1011 & 7,2 & 0,1471 & 0,0109 & \\
\hline 16. & 1500 & 1010 & 6,6 & 0,1575 & 0,0116 & \\
\hline 17. & 1200 & 1022 & 8,9 & 0,2142 & 0,0147 & \\
\hline 18. & 1000 & 1019 & 6,1 & 0,1383 & 0,0112 & \\
\hline 19. & 680 & 1024 & 6,0 & 0,1452 & 0,0119 & \\
\hline
\end{tabular}

also nur am 3. Tage eine Steigerung von $0,0635 \mathrm{~g} \overline{\mathrm{U}}-\mathrm{N}$ und $0,0017 \mathrm{~g}$ B-N in Summa $0,0652 \mathrm{~g}=$ ca. $6,6 \%$ der verfütterten N-Menge.

I. Auch hier wurde zurerst Thymus gegeben. Die Steigerung der Purinwerte (s. Tabelle III) betrug 0,0994 g $\overline{\mathrm{U}}-\mathrm{N}$ und $0,0020 \mathrm{~g} \mathrm{~B}-\mathrm{N}$, in Summa also $0,1014 \mathrm{~g}=$ ca. 8,5\% der verfütterten Purin-N-Menge.

Nach einigen Normaltagen wurde alsdann analog dem ersten Fall unser Xanthinpräparat in Wasser gelöst eingenommen. Die Gesamtsteigerung beträgt hier 0,1260 U-N und $0,0010 \mathrm{~B}-\mathrm{N}$, in Summa ca. $0,127 \mathrm{~g}=$ etwa $13 \%$ der verfütterten $\mathrm{N}$-Menge:

II. In der zweiten Periode, während der am 5. III. $2,1 \mathrm{~g}$ des Xanthinpräparates in derselben Weise appliziert wurden, trat eine Steigerung überhaupt nicht ein. 
Tabelle III. Selbstversuch.

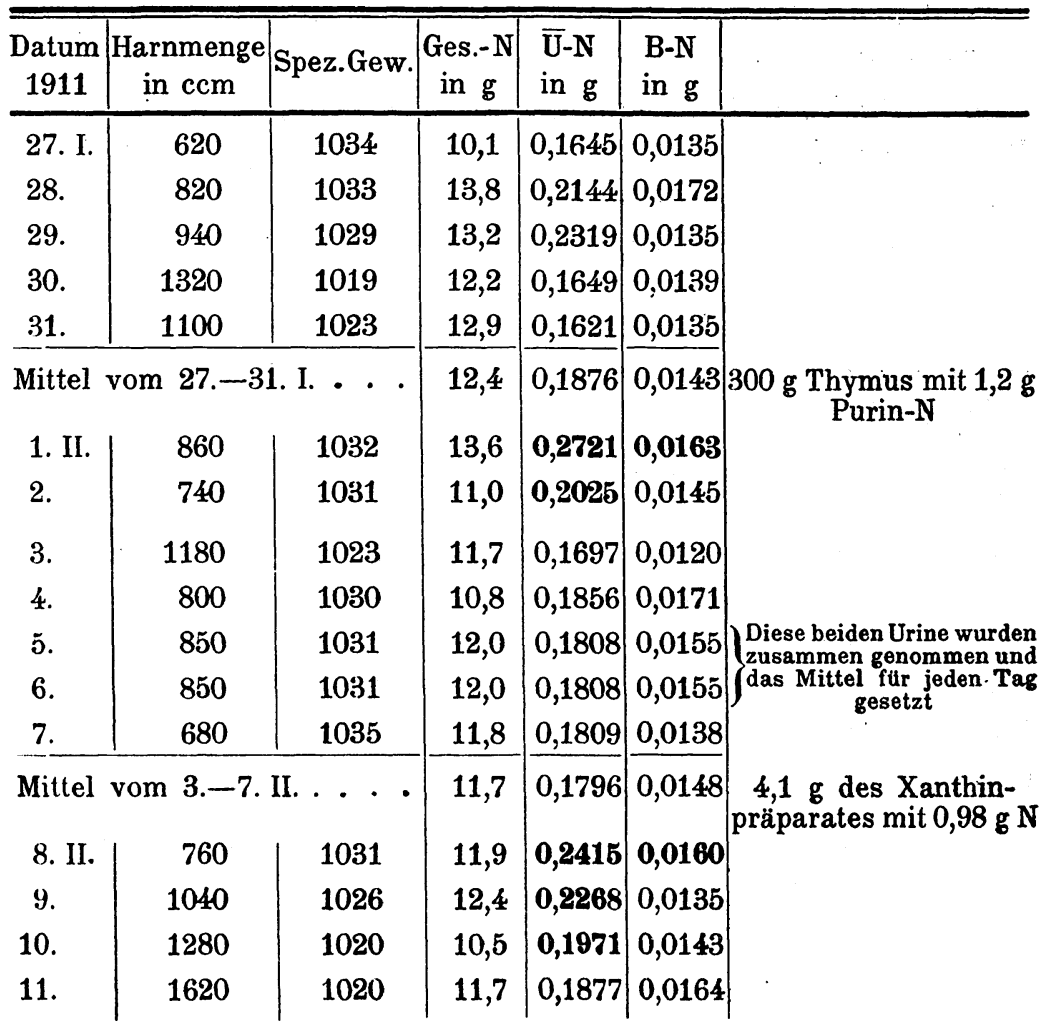

III. Da dieser Versager mehr oder weniger nur auf eine gestörte Resorption seitens des Darmes bezogen werden konnte und diese Auffassung in der ungenügenden Wasserlöslichkeit des Präparates ihre Unterstützung fand, so stellte ich mir nun zuerst die Aufgabe, wenn möglich eine Applikationsweise zu finden, mittels derer eine günstigere Ausnutzung zu erzielen wäre. Ich möchte an dieser Stelle Herrn Dr. Hans Fischer, dem Laboratoriumsvorstand, für seinen jederzeit freundlichst gegebenen Rat bestens danken. Ich nahm an, daß die kolloidale Natur des Xanthinniederschlages, der durch Essigsäure aus hochkonzentrierter alkalischer Lösung gefällt wird, der Resorption günstig sein müßte, besonders wenn man die vielleicht störende Wirkung der Salzsäure im Magen durch Darreichung von Natrium 
bicarbonicum paralysiert hätte. So wurde also für die folgenden Perioden die betreffende Menge des Präparates in wenig $\mathrm{NaOH}$ gelöst und unter starkem Umrühren Essigsäure bis zur leicht sauren Reaktion hinzugefügt. Dabei fiel ein gelblich weißer kolloidaler Niederschlag aus. Diese Suspension wurde nach Darreichung einer genügenden Menge von doppelkohlensaurem Natron vor dem Essen getrunken (s. Tab. IV!).

Tabelle IV. Selbstversuch.

\begin{tabular}{|c|c|c|c|c|c|c|}
\hline$\underset{1911}{\text { Datum }}$ & $\begin{array}{c}\text { Harnmenge } \\
\text { in } \mathrm{ccm}\end{array}$ & Spez.Gew. & $\left|\begin{array}{c}\text { Ges. }-\mathrm{N} \\
\text { in } \mathrm{g}\end{array}\right|$ & $\begin{array}{l}\overline{\mathrm{U}}-\mathrm{N} \\
\text { in } \mathrm{g}\end{array}$ & $\begin{array}{l}\mathrm{B}-\mathrm{N} \\
\text { in } \mathrm{g}\end{array}$ & \\
\hline 7. III. & 960 & 1024 & 11,5 & 0,1952 & 0,0155 & \multirow{10}{*}{$\begin{array}{l}2,05 \mathrm{~g} \text { des Xanthin- } \\
\text { präparates in essigs. } \\
\text { Fällung mit } 0,49 \mathrm{~g} \mathbf{N} \text {. }\end{array}$} \\
\hline 8. & 880 & 1029 & 10,5 & 0,1461 & 0,0119 & \\
\hline 9. & 680 & 1029 & 10,2 & 0,1333 & 0,0124 & \\
\hline 10. & 1120 & 1024 & 13,6 & 0,2062 & 0,0153 & \\
\hline 12. & 1000 & 1026 & 12,6 & 0,1880 & 0,0151 & \\
\hline 14. & 1160 & 1022 & 10,8 & 0,1721 & - & \\
\hline \multicolumn{3}{|c|}{ Mittel vom 7.-14. III. . • } & 11,5 & 0,1735 & 0,0140 & \\
\hline 15. III. & 1440 & 1028 & 13,5 & 0,2863 & 0,0146 & \\
\hline 16. & 1400 & 1022 & 9,8 & $0,2460 \mid$ & 0,0132 & \\
\hline 17. & 2440 & 1012 & 11,3 & 0,1810 & 0,0154 & \\
\hline
\end{tabular}

Das ist eine Steigerung von $0,185 \mathrm{~g} \overline{\mathrm{U}}-\mathrm{N}=\mathrm{ca} .38 \%$ der verfütterten N-Menge, die sich also hier auf die Harnsäure allein beziehen, während eine Steigerung des Basenstickstoffs überhaupt nicht auftrat.

$\mathrm{Zu}$ gleicher Zeit sollte durch Untersuchung der Faeces eventuell die der Resorption entgangene Menge wieder aufgefunden werden. Bei diesen Untersuchungen haben sich zwar keine völlig konstanten Basenwerte finden lassen, immerhin scheint mir, wie aus Tabelle V ersichtlich, eine gewisse Steigerung unverkennbar. Da ich vorher durch gleichzeitige Veräbreichung von Tierkohle festgestellt hatte, daß sich die Ausscheidung bis auf 3 Tage erstreckt, ergibt sich eine Gesamtsteigerung von ca. $0,132 \mathrm{~g}=$ etwa $27 \%$ der verfütterten $\mathrm{N}$-Menge, so daß in Urin und Kot zusammen ca. 65\% Purin-Stickstoff wiedergefunden werden konnten. Und hiervon traten also 
mehr als 2/5 im Kot auf, als ein Anteil, der den Darm unresorbiert passiert hat.

Tabelle V. Faeces.

\begin{tabular}{cc} 
Datum & \multicolumn{1}{c}{ B-N } \\
6. III. & 0,1140 \\
7. & 0,1260 \\
8. & 0,0840 \\
9. & 0,0725 \\
12. & 0,0958 \\
\hline Mittel ca. & 0,10 \\
15. Tag & $\mathbf{0 , 1 6 9 5}$ \\
16. : & 0,1050 \\
17. : & $\mathbf{0 , 1 6 2 4}$ \\
18. & 0,1040
\end{tabular}

-IV. Ein charakteristisches Beispiel von der großen Bedeutung der Resorptionsverhältnisse auf die Ausscheidung im Urin gibt der nächste Versuch. Hier sollte am 21. III. eine doppelt so große Menge des Präparates in 5 maliger Dosis nach der gleichen Methode verabreicht werden. Schon beim vierten Male trat so erheblicher Widerwillen auf, daß auf die letzte Dosis völlig verzichtet werden mußte und nur eine Gesamtmenge von $3,2 \mathrm{~g}$ mit $0,75 \mathrm{~g} \mathrm{~N}$ eingenommen werden konnte. In der Folge stellte sich leichter Durchfall ein; dementsprechend findet sich auch nur am 22. eine geringe Steigerung von etwa $0,045 \mathrm{~g} \overline{\mathrm{U}}-\mathrm{N}$ und $0,004 \mathrm{~g} \mathrm{B-N}$, das ist in Summa ca. 0,05= nicht ganz 7\%. Im Kot konnte die der Resorption offenbar entgangene Menge hierbei nicht wieder gefunden werden. Die Erklärung dafür ist m. E. diese: Wie Schittenhelm ${ }^{1}$ ) gezeigt hat, unterliegen die Purinbasen des Kotes, und zwar sowohl die physiologischen wie die experimentell zugesetzten, bei der Fäulnis einer bakteriellen oder fermentativen Zerstörung. Und ein Opfer dieser durch die Diarrhöe noch verstärkten Fäulnis sind offenbar auch die der Resorption entgangenen Xanthinmengen in unserem Falle geworden. Ich will nicht unerwähnt lassen, daß von mir selbst angestellte Fäulnisversuche mit Dünndarminhalt und Kot, die nach Zusatz von Xanthin 48 Stunden

1) Schittenhelm, Diese Zeitschrift, Bd. 39, S. 199, 1903. 
bei $37^{\circ}$ C. gehalten wurden, die Resultate Schittenhelms bestätigt haben.

V. Im Gegensatz zu diesem letzten Versuch wurde jetzt die geringe Menge von 1,2 g des Präparates, nach derselben Methode gefällt, in 3 maliger Dosis von je $0,4 \mathrm{~g}$ gegeben. Tabelle VI zeigt mit der Wiedergewinnung von $76 \%$ eine bisher ungewöhnlich hohe Ausnutzung.

Tabelle VI. Selbstversuch.

\begin{tabular}{l|c|c|r|r|r|r}
\hline $\begin{array}{l}\text { Datum } \\
1911\end{array}$ & $\begin{array}{c}\text { Harnmenge } \\
\text { in ccm }\end{array}$ & Spez.Gew. & $\begin{array}{r}\text { Ges-N. } \\
\text { in g }\end{array}$ & $\begin{array}{c}\overline{\mathrm{U}}-\mathrm{N} \\
\text { in g }\end{array}$ & $\begin{array}{l}\mathrm{B}-\mathrm{N} \\
\text { in g }\end{array}$ & \\
\hline 23. III. & 1080 & 1028 & 10,3 & 0,1569 & 0,0110 & \\
24. & 1280 & 1019 & 11,4 & 0,1680 & - & \\
26. & 1320 & 1023 & 11,1 & 0,1848 & 0,0125 & \\
28. & 960 & 1024 & 8,3 & 0,1559 & 0,0148 & \\
\hline Mittel vom $23 .-28$. & III. . . & 10,3 & 0,1664 & 0,0128 & $\begin{array}{c}1,2 \text { g des Xanthin- } \\
\text { präparates in essigs. }\end{array}$ \\
29. III. & 1080 & 1030 & 12,4 & $\mathbf{0 , 2 6 4 6}$ & $\mathbf{0 , 0 2 1 2}$ & Fällung mit 0,288 g N. \\
30. & 1160 & 1025 & 11,4 & $\mathbf{0 , 2 4 5 2}$ & verloren & \\
31. & 1440 & 1022 & 10,5 & $\mathbf{0 , 1 9 2 5}$ & $\mathbf{0 , 0 1 6 6}$ & \\
1. IV. & 2240 & 1012 & 11,3 & 0,1788 & $\mathbf{0 , 0 1 8 0}$ &
\end{tabular}

Das ist eine Steigerung von $0,2031+0,0174$, in Summa mehr als $0,22 \mathrm{~g}$ $=$ über $76 \%$ der verfütterten N-Menge.

In diesen Ergebnissen schien mir der Weg zum Verständnis der Differenzen in den verschiedenen Literaturangaben und in meinen eigenen Versuchen klar gewiesen. Da es sich beim Xanthin bekannterweise um einen schwer resorbierbaren Körper handelt, so konnten jene Schwankungen sehr wohl auf die den verschiedensten Einflüssen unterworfenen Resorptionsverhältnisse bezogen werden. Volles Licht mußte in diese Dinge zu bringen sein, wenn es gelänge, das Präparat mit Ausschließung der Darmresorption dem Organismus einzuverleiben. Mir sind selbstverständlich sehr wohl jene Einwände bekannt, die gelegentlich der parenteralen Harnsäureversuche, wie sie mehrfach $^{1}$ ) angestellt worden sind, von verschiedenen Seiten,

1) Cf. Burian u. Schur, Arch. f. d. ges. Physiol., Bd. 87, S. 326, 1901. - Ibrahim und Soetbeer, Diese Zeitschrilt, Bd. 35, S. 1, 1902. 
hauptsächlich von Schittenhelm und Brugsch, erhoben wurden und auch heute noch aufrecht erhalten werden. Aber ich erinnere daran, daß es sich hier immer um subcutane resp. intramuskuläre Injektionen gehandelt hat, und verweise auf jenen klassischen Versuch von Umber ${ }^{1}$ ) und seine klaren und eindeutigen Begründungen, wenn er als erster Harnsäure direkt durch intravenöse Injektion in die Blutbahn bringt. Es ist bekannt, daß es ihm gelang, beim Gesunden in einem Fall $80 \%$ im Laufe von 4 Tagen, im 2. Fall $94,6 \%$ im Laufe von 2 Tagen wiederzugewinnen. Dieser Umber sche Versuch wurde für mich maßgebend bei der weiteren Untersuchung des Xanthinabbaues. Ich hoffte mit einer analogen Anordnung ein letztes Resultat eindeutig zu gewinnen. Wie bei Umber sollte das Xanthin in Piperazin gelöst injiziert werden. Es versteht sich von selbst, daß ein solcher Versuch, der bisher, so viel ich weiß, noch niemals unternommen worden ist, und für den ich auch bisher keine Analogien unter den Tierexperimenten finden konnte, unter den größten Kautelen angestellt werden mußte. Ich schaltete deshalb hier zunächst ein Kaninchen experiment ein. Das etwa $4 \mathrm{~kg}$ schwere männliche Kaninchen, dessen Urin zweimal am Tage mittels Katheter gewonnen wurde, erhielt eine Menge von 0,4 $\mathrm{g}$ des Präparates mit $0,096 \mathrm{~g} \mathrm{~N}$ in Piperazin gelöst körperwarm in die Ohrvene eingespritzt. Leider gelang keine völlig quantitative Einverleibung, und ein kleiner Rest im Glase zeigte noch deutliche Xanthinreaktion. Obwohl dieser Versuch natürlich in erster Linie unternommen war, um lediglich die Unschädlichkeit resp. die Giftigkeit des Präparates zu erproben, so durfte doch eine Bestimmung der Purinwerte nicht unterbleiben.

Im Gegensatz zu anderen Autoren, wie Schittenhelm und Bendix, ${ }^{2}$ ) Mendel und Lyman ${ }^{3}$ ) finde ich auch für die

- Wiechowski, Arch. f. exp. Pathol. u. Pharmak., Bd. 60, S. 185, 1909.

- Benzcûr, Z. f. exper. Pathol., Bd. 7, S. 339, 1909. April 1910.

1 Umber und Retzlaff, Deutsch. Kongr. f. innere Med. Wiesbaden,

2) Schittenhelm u. Bendix, Diese Zeitschrift, Bd. 42, S. 461.

${ }^{3}$ ) Mendel und Lyman, 1. c. 
Normaltage eine deutliche Ausscheidung von Harnsäure und Purinbasen, und zwar sind meine Durchschnittswerte etwa $0,010 \mathrm{~g}$ für $\overline{\mathrm{U}}-\mathrm{N}$ und $0,008 \mathrm{~g}$ für $\mathrm{B}-\mathrm{N}$.

Am Tage der Injektion zeigt nun der Urin Erhöhungen auf $0,0477 \mathrm{~g} \overline{\mathrm{U}}-\mathrm{N}$ und $0,0398 \mathrm{~g}$ B-N, die am nächsten Tage bereits abgeklungen sind; das sind also Steigerungen um zirka $0,038 \mathrm{~g} \overline{\mathrm{U}}-\mathrm{N}$ und $0,032 \mathrm{~g}$ B-N, in Summa $0,07 \mathrm{~g}, \mathrm{~d}$. h. eine Wiedergewinnung von über $75 \%$.

Auf das Verhältnis von Harnsäure zu Basen wird nachher noch kurz einzugehen sein. Das Kaninchen ertrug die Injektion tadellos, zeigte weder allgemeine noch wesentliche lokale Störungen. Auf dieser Basis konnte nun an den Selbstversuch gegangen werden, nachdem noch ein peroraler Versuch mit dem in Piperazin gelösten Präparat vorausgeschickt war, um auch bei dieser Darreichungsmethode einen Vergleichswert für den Einfluß der Resorption zu gewinnen.

Tabelle VII. Selbstversuch.

\begin{tabular}{l|c|c|c|c|c|c}
\hline $\begin{array}{c}\text { Datum } \\
1911\end{array}$ & $\begin{array}{c}\text { Harnmenge } \\
\text { in ccm }\end{array}$ & Spez.Gew. & $\begin{array}{c}\text { Ges.-N } \\
\text { in g }\end{array}$ & $\begin{array}{c}\overline{\mathrm{U}}-\mathrm{N} \\
\text { in g }\end{array}$ & $\begin{array}{c}\mathrm{B}-\mathrm{N} \\
\text { in g }\end{array}$ & \\
\hline 7. VI. & 700 & 1029 & 10,9 & 0,1553 & 0,0127 & \\
8. & 900 & 1028 & 13,5 & 0,1853 & 0,0139 & \\
9. & 900 & 1026 & 13,0 & 0,1600 & 0,0139 & \\
\hline Mittel vom $7 .-9$. & VI. . . . & 12,5 & 0,1669 & 0,0135 & 1,0 g des Xanthin- \\
präparates in Piperazin \\
10. VI. & 1200 & 1021 & 14,0 & $\mathbf{0 , 2 2 4 3}$ & $\mathbf{0 , 0 1 6 0}$ & per o s mit 0,24 g N. \\
11. & 1800 & 1012 & 12,0 & $\mathbf{0 , 1 9 1 5}$ & $\mathbf{0 , 0 1 5 8}$ & \\
12. & 1000 & 1026 & 13,4 & 0,1799 & 0,0133 & \\
13. & 1400 & 1015 & 13,0 & 0,1578 & 0,0147 &
\end{tabular}

Das ist eine Steigerung von $0,0951+0,0048$, in Summa 0,10=ca. $42 \%$ der verfütterten $\mathrm{N}$-Menge.

VII. Am 14. VI. wurde eine Menge von $0,5 \mathrm{~g}$ des Präparates in einer Piperazinlösung, die $2 \mathrm{~g}$ Piperazin auf $60 \mathrm{ccm}$ Wasser enthielt, körperwarm in Kochsalzlösung eingeschaltet in die linke Cubitalvene injiziert. Die Injektion selbst 
verlief ohne besondere Störung oder sonstige Sensationen. Beobachtungen, wie sie Umber für die Harnsäureinjektion berichtet, konnten nicht gemacht werden, schon deshalb, weil natürlicherweise das autosuggestive Moment nicht auszuschalten war. Ehe ich auf eine Darstellung der Resultate eingehe, muß berichtet werden, daß sich im Anschluß an diesen Versuch doch zwei Erscheinungen einstellten, von denen die eine in wahrscheinlichem, die andere in zweifellosem Zusammenhang mit der Injektion selbst steht. Nachdem der Urin bereits am zweiten Tage auf die normalen Werte zurückgekehrt war, die gesamte Ausscheidung also an einem Tage stattgefunden hatte, trat am dritten Tage eine unverkennbare Steigerung und am vierten Tage eine kolossale Erhöhung beider Werte, besonders exzeptionellerweise des Basenwertes auf. Und diese Steigerung war begleitet von einem ganz plötzlich und ziemlich heftig einsetzenden Schmerzanfall im rechten Knie, der nachts nach einer ganz mäßigen Strapaze durch Tanzen auftrat, keine Rötung, aber ganz leichte Schwellung und deutliche lokale Temperatursteigerung erkennen ließ. Dieser Anfall hielt langsam abnehmend am nächsten Tage (dem 18. VI.) an und war am 19. völlig geschwunden. Ich habe für diese Erscheinung, bei der zum mindesten die Kongruenz von Purinsteigerung und Gelenkentzündung auffällt, keine Erklärung, bemerke übrigens, daß bei mir zurzeit das rechte Bein infolge einer postdiphtherischen Lähmung und daran anschließenden Muskelatrophie einen locus minoris resistentiae darstellte.

Klarer ist die zweite Erscheinung. Eine mäßige Schmerzhaftigkeit am linken Arm wurde anfangs von mir nicht weiter beachtet und lediglich auf den Einstich bezogen; als ich jedoch nach ein paar Tagen zufällig den Arm abtastete, zeigte sich eine Thrombose der Vena cephalica, die bis zum Deltøideus verfolgbar war und nach unten etwas über die Ellenbeuge hinausreichte. Es hat sich also herausgestellt, daß Xanthin für den menschlichen Organismus zu giftig ist und eine Wiederholung des intravenösen Versuches aus diesem Grunde unterbleiben mußte. Immerhin hat dieser Versuch zu interessanten Werten geführt, in denen ich wohl unbedingt eine Bestätigung 
meiner Auffassung sehen darf. Auf die oben gegebene Zahlenreihe (Tabelle VII) folgen als Werte des Injektionstages:

\begin{tabular}{|c|c|c|c|c|c|c|}
\hline $\begin{array}{c}\text { Datum } \\
1911\end{array}$ & $\left|\begin{array}{c}\text { Harnmenge } \\
\text { in } \mathrm{ccm}\end{array}\right|$ & Spez. Gew. & $\mid \begin{array}{c}\text { Ges.- N } \\
\text { in } \mathrm{g}\end{array}$ & $\begin{array}{l}\overline{\mathrm{U}}-\mathrm{N} \\
\text { in } \mathrm{g}\end{array}$ & $\begin{array}{l}\mathrm{B}-\mathrm{N} \\
\text { in } \mathrm{g}\end{array}$ & \\
\hline Mittel & der Vortage & e . . & 12,5 & 0,1669 & 0,0135 & \multirow{6}{*}{$\begin{array}{l}\text { nachts zwischen } 17 . / 18 \\
\text { Schmerzanfallim r.Knie }\end{array}$} \\
\hline 1ว̆. VI. & 1550 & 1021 & 15,2 & 0,2647 & 0,0217 & \\
\hline 16. & 1800 & 1012 & 11,9 & 0,1726 & 0,0126 & \\
\hline 17. & 1300 & 1024 & 13,9 & 0,2038 & 0,0173 & \\
\hline 18. & 1050 & 1026 & 13,8 & 0,2499 & 0,0257 & \\
\hline 19. & 600 & 1028 & 11,4 & $|0,1693|$ & $\mid 0,0113$ & \\
\hline
\end{tabular}

Das ist also eine Steigerung von $0,0978+0,0082$, in Summa $0,0160 \mathrm{~g} \mathrm{~N}$ von der einverleibten Xanthinmenge mit einem $\mathrm{N}$-Gehalt von 0,12 g.

Auf Prozente berechnet macht das eine Wiedergewinnung von ca. $89 \%$, die sich mit $\mathbf{8 1 , 5} \%$ auf Harnsäure und $7 \%$ auf die Basen, d. h. wohl unverändertes Xanthin, verteilen. Ich glaube, daß dieses Resultat ein gutes Analogon zu den Umberschen Versuchen darstellt, und darf wohl meine Gesamtresultate zusammenfassen in dem Satz: Mit einer hohen Wahrscheinlichkeit wird das gesamte in den Stoffwechselkreislauf des Menschen gelangte Xanthin quantitativ ohne Sprengung des Purinringes wieder ausgeschieden, wobei die Hauptmenge zu Harnsäure als Endprodukt oxydiert wird, ein kleiner Rest unverändert den Organismus passiert. Bei sämtlichen peroralen Darreichungen des Xanthins kann anderseits immer nur eine teilweise Aufnahme in die Zirkulation erwartet werden, die in jedem Einzelfalle von den verschiedensten und zufälligsten Resorptionsbedingungen abhängig ist.

Von dieser Grundlage aus mag noch ein Blick auf die Zahlen geworfen werden, die an einem Gichtkranken gewonnen wurden.

M. B., Dienstmann, 60 Jahre, Körpergewicht 65 Kilo. Hat gedient. Früher Potus $(81$ Bier). Vor 2 Jahren Gichtanfall in Händen und Füßen. Jetzt neuer Anfall in beiden Füßen. Zahlreiche Tophi, Alb. u. Sacch. negativ. Während der Untersuchungsperiode kein neuer Anfall und weder nach Thymus noch nach Xanthin die geringste Verschlimmerung im Befinden. 
Der Patient, der schon längere Zeit auf purinfreie Diät gesetzt war, erhielt am 7. Dezember 1910 zu seiner gewöhnlichen Kost $300 \mathrm{~g}$ Thymus. Am ersten Tage trat weder für die Harnsäure, noch für die Basen eine Veränderung auf. Am zweiten, dritten und vierten Tage stieg der Gehalt von B-N ein geringes an, um am nächsten Tage auf die Norm zurückzukehren. Die Harnsäure zeigte nur am vierten Tage, das ist dem dritten Tage der Basensteigerung eine Erhöhung, um dann ebenfalls zur Norm zurückzukehren (s. Tabelle VIII), und zwar zeigt sich im ganzen eine Steigerung von $0,0282 \mathrm{~g} \overline{\mathrm{U}}-\mathrm{N}+0,0054 \mathrm{~g}$ B-N, in Summa $0,0336 \mathrm{~g}=\mathbf{2 , 8} \%$ des verfütterten Thymus-N.

Nach drei weiteren Normaltagen wurde eine Menge von $4,1 \mathrm{~g}$ unseres Xanthinpräparates in wässeriger Lösung per os gegeben. Jetzt trat bereits am nächsten Tage sowohl eine Steigerung der Harnsäure- wie der Basenausscheidung auf, von denen die erstere 3 Tage anhielt, die letztere 2 Tage. Und zwar beträgt die Steigerung des

$\overline{\mathrm{U}}-\mathrm{N}$ 0,0600, die des B-N 0,0040, d. i. in Summa 0,064 = 6,5 $\%$ der verfütterten $\mathrm{N}$-Menge.

Die geringe Steigerung der Purinwerte nach dem Thymus entspricht den bekannten Retentionsverlusten. Die Steigerung nach dem Xanthin ist mit 6,5\% zwar wesentlich höher, als die nach der Thymusdarreichung, bleibt aber in entsprechender Weise zurück hinter dem analogen Wert bei mir selbst $(13 \%)$. Obwohl in allen Fällen die Ausbeute des Xanthins nach der einfachen Darreichung in Wasser bei einer Menge, die wenigstens ganz ungefähr dem Stickstoffgehalt von $300 \mathrm{~g}$ Thymus entspricht, besser war als nach Thymus, so ist doch diese Ausscheidung als Ausdruck der wechselnden und unsicheren Resorptionsmenge gar zu unzuverlässig, als daß sich jene anfangs erwähnte Hoffnung auf Gewinnung eines brauchbaren Ersatzes für Thymus im Xanthin erfüllt hätte.

- Ich möchte diese Betrachtung nicht abschließen, ohne auf eine Erscheinung hinzuweisen, die im Hinblick auf mancherlei Bemerkungen in der Literatur und auch jetzt aktuelle Probleme von besonderem Interesse erscheint. Wenn ja auch bekannter- 
Tabelle VIII. Gichtiker M. B.

\begin{tabular}{|c|c|c|c|c|c|c|}
\hline $\begin{array}{c}\text { Datum } \\
1910\end{array}$ & $\left|\begin{array}{c}\text { Harnmenge } \\
\text { in } \mathrm{ccm}\end{array}\right|$ & Spez.Gew. & $\left|\begin{array}{c}\text { Ges. }-N \\
\text { in } g\end{array}\right|$ & $\begin{array}{l}\bar{U}-\mathrm{N} \\
\text { in } g\end{array}$ & $\begin{array}{l}\mathrm{B}-\mathrm{N} \\
\text { in } \mathrm{g}\end{array}$ & \\
\hline 2.XII. & 1710 & 1010 & 8,1 & 0,0593 & $|0,0102|$ & \multirow{13}{*}{$\begin{array}{c}300 \mathrm{~g} \text { Thymus mit } 1,2 \mathrm{~g} \\
\text { Purin-N }\end{array}$} \\
\hline 3. & 1460 & 1008 & 4,9 & 0,0225 & 0,0031 & \\
\hline ธ. & 1850 & 1007 & 8,0 & 0,0492 & 0,0071 & \\
\hline 6. & 2600 & 1008 & 10,0 & 0,0582 & 0,0073 & \\
\hline 7. & 1780 & 1007 & 7,7 & 0,0554 & 0,0081 & \\
\hline \multicolumn{3}{|c|}{ Mittel vom 2.-7. XII. . . • } & 7,7 & 0,0500 & 0,0071 & \\
\hline 8.XII. & 1600 & 1010 & 7,8 & 0,0465 & 0,0067 & \\
\hline 9. & 1710 & 1007 & 6,6 & 0,0575 & 0,0090 & \\
\hline 10. & 1710 & 1007 & 7,4 & 0,0569 & 0,0081 & \\
\hline 11. & 1520 & 1010 & 9,8 & 0,0782 & 0,0096 & \\
\hline 13. & 1680 & 1009 & 7,3 & 0,0535 & 0,0071 & \\
\hline 14. & 1260 & 1011 & 8,6 & 0,0507 & 0,0066 & \\
\hline 15. & 1650 & 1008 & $6, \tilde{5}$ & 0,0433 & 0,0058 & \\
\hline \multicolumn{3}{|c|}{ Mittel vom 13.-15. XII. . · } & 7,5 & 0,0500 & 0,0065 & \multirow{5}{*}{$\begin{array}{c}\mathbf{4 , 1} \mathrm{g} \text { des Xanthin- } \\
\text { präparates mit } \\
0,98 \mathrm{~g} \mathrm{~N} .\end{array}$} \\
\hline 16. XII. & 2160 & 1007 & 8,8 & 0,0756 & 0,0091 & \\
\hline 17. & 1740 & 1007 & 8,0 & 0,0706 & 0,0079 & \\
\hline 18. & 1660 & 1009 & 7,1 & 0,0639 & 0,0058 & \\
\hline 19. & 1300 & 1010 & 5,6 & 0,0505 & $|0,0068|$ & \\
\hline
\end{tabular}

maßen das Verhältnis von Harnsäure zu Purinbasen, oder anders ausgedrückt der Prozentanteil der Basen am Gesamtpurin-N im Urin des purinfrei ernährten Menschen gewissen Schwankungen unterworfen ist, so läßt sich doch im allgemeinen, ${ }^{1}$ ) wie für unseren Fall der physiologische Anteil des Basenstickstoffs als ungefähr $7 \%$ des Gesamtpurin-N angeben. Und nun zeigt es sich, daß die Xanthinausscheidung des Injektionsversuches in einem annähernden Verhältnis, nämlich mit einem Basenanteil von ca. 7,7\% stattgefunden hat. Dieser Hinweis findet eine interessante Unterstützung in der Betrachtung der Kaninchenwerte. Da dort jenes Verhältns ein anderes ist und zwar der Anteil des Basen-N am Gesamtpurin-N ungefähr 44\% beträgt,

1) cf. Julie Hefter, Inaug.-Diss. München 1911. 
so zeigt sich auch bei der Verarbeitung des Xanthins ein ganz anderes Bild, als wir es beim Menschen gefunden haben. Auch dort entspricht der Basenanteil mit ca. 43,6\% ungefähr dem physiologischen Verhältns.

Ich möchte an diese Purinstoffwechseluntersuchung noch eine kleine Versuchsreihe anschließen, die der anfangs skizzierten Frage vom Abbau der methylierten Xanthine, im besonderen dem Problem gewidmet war, ob aus Coffein im Organismus Harnsäure entsteht. Bekanntlich leugnet die Mehrzahl der Autoren (so Minkowski, 1) Schutzkwer, ${ }^{2}$ ) Krüger und Schmid, ${ }^{3}$ ) Schmid ${ }^{4}$ ) u. a.), die Entstehung von Harnsäure aus Coffein. Im Gegensatz zu ihnen gelangen aber Besser, ${ }^{5}$ ) ferner $\mathrm{Axisa}^{6}$ ) $\mathrm{zu}$ einer Bejahung der Frage, zu der sich bei Schittenhelm ${ }^{7}$ ) im Hundeversuch ein Analogon in der Vermehrung der Allantoinausscheidung nach Coffein findet.

Es darf nicht verschwiegen werden, daß nach diesen Publikationen Schmid $^{8}$ ) mit Organversuchen noch einmal zu negativen Resultaten kommt und so die ältere Anschauung, wenn auch nicht sehr überzeugend, von neuem begründet.

Noch innerhalb der purinfreien Diät nahm ich zuerst am 20. VI. per os $1,5 \mathrm{~g}$ Coffein. puri mit $0,433 \mathrm{~g} \mathrm{~N}$ in sechsmaliger Dosis ein (s. Tabelle IX). Wenn auch, wie längst bekannt, der Hauptanteil in der Steigerung des Purin-N nach Darreichung von Coffein im Basengemisch - hier mit insgesamt $0,085 \mathrm{~g}$ - wiedergefunden wird, so läßt sich doch eine deutliche Steigerung auch des Harnsäurewertes - von ca. 0,07 g in diesem Falle nicht verkennen. Im ganzen stellt diese Steigerung von $0,155 \mathrm{~g}$ eine Wiedergewinnung von nicht ganz $36 \%$ der verfütterten $\mathrm{N}$-Menge dar. Bemerkenswert erscheinen mir nun die Resultate des zweiten Versuches, bei den am 29. VI.

1) Minkowski, Arch. f. exp. Pathol. u. Pharm., Bd. 41, S. 406, 1898.

2) Schutzkwer, Inaug.-Diss. Königsberg 1882.

3) Krüger und Schmid, Diese Zeitschrift, Bd. 32, S. 104, 1900.

4) Schmid, Deutsch. Arch. f. klin. Med., Bd. 77, S. 505, 1903.

5) Besser, Ther. d. Gegenw., Bd. 50, S. 321, 1909.

6) Axisa, Zentralbl. f. innere Med., Bd. 31, S. 113, 1910.

7) Schittenhelm, Ther. Monatsh., Bd. 24, S. 113, 1910.

8) Schmid, Diese Zeitschrift, Bd. 67, S. 155, 1910. 
eine Menge von 3,2 $\mathrm{g}$ Coffein. natr.-citr., gelöst in ca. $50 \mathrm{ccm}$ Wasser, wieder in sechsfacher Dosis subcutan injiziert wurde. Diese Menge von 3,2 $\mathrm{g}$ des Doppelsalzes entspricht genau 1,5 $\mathrm{g}$ reinen Coffeins mit 0,433 g N (s. Tabelle IX). Es braucht nicht nochmals darauf hingewiesen $z u$ werden, daß wiederum der Hauptanteil der Steigerung mit 0,063 g im Basengemisch steckt. Wenn nun auch der Harnsäurewert mit 0,1829 das Mittel ein wenig überschreitet, so stellt diese Zahl doch einen Wert dar, der durchaus innerhalb der Schwankungsgrenze liegt. Gleichzeitig fällt auf, daß bei dieser parenteralen Applikation nur etwa 14,5\% des Stickstoffs in Form von Purin- $\mathrm{N}$ im Urin wieder erscheinen. Nun muß aber besonders darauf hingewiesen werden, daß in beiden Fällen eine erhebliche Steigerung des Gesamtstickstoffes auftritt, und daß die Urinmenge eine starke Vermehrung - im ersten Fall bis auf das dreifache - erfährt. Es muß also daran gedacht werden, daß auch die Steigerung der Harnsäure lediglich eine Folge der diuretischen Coffeinwirkung darstellt, sodaß irgend welche Schlüsse auf eine Harnsäurebildung aus Coffein in unserem Falle nicht gezogen werden dürfen.

Tabelle IX. Selbstversuch.

\begin{tabular}{|c|c|c|c|c|c|c|}
\hline $\begin{array}{c}\text { Datum } \\
1911\end{array}$ & $\begin{array}{c}\text { Harnmenge } \\
\text { in } \mathrm{ccm}\end{array}$ & Spez.Gew. & $\begin{array}{c}\text { Ges.- } N \\
\text { in } g\end{array}$ & $\begin{array}{l}\bar{U}-\mathrm{N} \\
\text { in } \mathrm{g}\end{array}$ & $\begin{array}{l}\mathrm{B}-\mathrm{N} \\
\text { in } \mathrm{g}\end{array}$ & \\
\hline 19. VI. & 600 & 1028 & 11,4 & 0,1693 & 0,0113 & \multirow{8}{*}{1,5 g Coff. pur. mit } \\
\hline 20. & 900 & 1027 & 11,4 & 0,1953 & 0,0139 & \\
\hline 21. & 2600 & 1010 & 15,1 & 0,2430 & 0,0664 & \\
\hline 22. & 740 & 1024 & 10,6 & 0,1456 & 0,0466 & \\
\hline 23. & 740 & 1033 & 13,6 & 0,1678 & 0,0135 & \\
\hline 24. & 940 & 1029 & 13,5 & 0,1750 & $0,0,32$ & \\
\hline 25. & 700 & 1030 & 8,6 & 0,1838 & 0,0137 & \\
\hline 26. & 850 & 1028 & 12,2 & 0,1815 & 0,0149 & \\
\hline 29. & 900 & 1026 & 11,3 & 0,1600 & 0,0120 & \multirow{5}{*}{$\begin{array}{l}3,2 \mathrm{~g} \text { Coff. natr. citr. } \\
(=1,5 \mathrm{~g} \text { Coff. pur. }) \text { mit } \\
0,433 \mathrm{~g} \mathrm{~N} \text { subcutan }\end{array}$} \\
\hline 30. & 1300 & 1024 & 14,7 & 0,1829 & 0,0582 & \\
\hline 1. VII. & 600 & 1028 & 12,3 & 0,1659 & 0,0286 & \\
\hline 2. & 1125 & 1022 & 14,9 & 0,1654 & 0,0158 & \\
\hline 3. & 1000 & 4022 & 12,4 & 0,1533 & 0,0119 & \\
\hline
\end{tabular}


Bemerkenswert ist, daß auch bei dem Gichtiker nach Coffein neben der Basensteigerung eine bedeutende Erhöhung der Harnsäureausscheidung auftrat, und zwar wie nicht verwunderlich verzögert erst am 3. Tage. Aber auch hier zeigt sich mit der gleichzeitigen Erhöhung des Gesamtstickstoffs ein dem Selbstversuch entsprechendes Bild, und auch hier muß an eine lediglich diuretische Steigerung der Harnsäure gedacht werden.

Tabelle X. Gichtiker M. B.

\begin{tabular}{l|c|c|c|c|c|c}
\hline \hline $\begin{array}{c}\text { Datum } \\
1910\end{array}$ & $\begin{array}{c}\text { Harmmenge } \\
\text { in ccm }\end{array}$ & Spez.Gew. & $\begin{array}{c}\text { Ges.-N } \\
\text { in g }\end{array}$ & $\begin{array}{c}\overline{\mathrm{U}}-\mathrm{N} \\
\text { in g }\end{array}$ & $\begin{array}{c}\mathrm{B}-\mathrm{N} \\
\text { in g }\end{array}$ & \\
\hline $19 . \mathrm{XII}$. & 1300 & 1010 & 5,6 & 0,0505 & 0,0068 & \\
20. & 2410 & 1007 & 7,8 & 0,0565 & 0,0076 & \\
21. & 1600 & 1007 & 6,8 & 0,0493 & 0,0062 & \\
22. & 1600 & 1009 & 6,5 & 0,0465 & $\mathbf{0 , 0 0 6 2}$ & $\mathbf{1 , 5}$ g Coff. pur. mit \\
23. & 1660 & 1009 & 6,5 & 0,0610 & $\mathbf{0 , 0 1 3 4}$ & \\
24. & 960 & 1008 & 4,2 & 0,0494 & $\mathbf{0 , 0 1 2 1}$ & \\
25. & 1580 & 1009 & $\mathbf{8 , 4}$ & $\mathbf{0 , 0 7 0 2}$ & $\mathbf{0 , 0 1 2 2}$ & \\
26. & 1700 & 1008 & $\mathbf{7 , 6}$ & 0,0506 & $\mathbf{0 , 0 0 8 3}$ &
\end{tabular}

Das ist eine Steigerung von ca. 0,02 + 0,02, in Summa 0,04 $\mathbf{g}$ $=$ etwas über $9 \%$ der verfütterten $\mathrm{N}$-Menge.

Zum Schlusse freue ich mich, Herrn Prof. Friedrich Müller für die Stellung der Arbeit und sein liebenswürdiges Interesse, Herrn Prof. Neubauer für freundliche Beratung herzlich danken zu dürfen. 